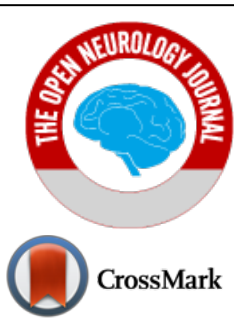

REVIEW ARTICLE

\title{
An Overview of Studies Demonstrating that ex vivo Neuronal Networks Display Multiple Complex Behaviors: Emergent Properties of Nearest-Neighbor Interactions of Excitatory and Inhibitory Neurons.
}

\author{
Thomas B. Shea ${ }^{1, *}$ \\ ${ }^{1}$ Laboratory for Neuroscience, Department of Biological Sciences, University of Massachusetts Lowell, Lowell, MA 01854, USA
}

\begin{abstract}
:
The responsiveness of the human nervous system ranges from the basic sensory interpretation and motor regulation to so-called higher-order functions such as emotion and consciousness. Aspects of higher-order functions are displayed by other mammals and birds. In efforts to understand how neuronal interaction can generate such a diverse functionality, murine embryonic cortical neurons were cultured on Petri dishes containing multi-electrode arrays that allowed recording and stimulation of neuronal activity. Despite the lack of major architectural features that govern nervous system development in situ, this overview of multiple studies demonstrated that these 2-dimensional ex vivo neuronal networks nevertheless recapitulate multiple key aspects of nervous system development and activity in situ, including density-dependent, the spontaneous establishment of a functional network that displayed complex signaling patterns, and responsiveness to environmental stimulation including generation of appropriate motor output and long-term potentiation. These findings underscore that the basic interplay of excitatory and inhibitory neuronal activity underlies all aspects of nervous system functionality. This reductionist system may be useful for further examination of neuronal function under developmental, homeostatic, and neurodegenerative conditions.
\end{abstract}

Keywords: Neuronal culture, Multi-electrode arrays, Synaptic signaling, Inhibitory neurons, Excitatory neurons, Nervous system development.

\begin{tabular}{l|l|l|l} 
Article History & Received: September 09, 2020 & Revised: January 04, 2021 & Accepted: January 14, 2021
\end{tabular}

\section{INTRODUCTION}

Integration of complex environmental information and generation of appropriate responses are mediated by neurons [1]. Dendrites collect sensory input, which is integrated at the cell body and summarized as a "fire/no fire" action via the axon and associated synapse (Fig. 1A). The simplest response, such as that of a hydra, consists of sensory input motor output, mediated by simple neuronal connections of primitive ganglia [2]. Notably, however, evidence has been presented that multifunctional neurons of the hydra may have functioned essentially as neuronal stem cells that yielded sensory, motor, and interneurons as well as neurosecretory cells observed in more complex nervous systems [3]. It has been suggested that the simple evolutionary development of "integration centers" on opposite ends of a simple nerve net ultimately generated the bilaterian nerve cord and brain of complex animals [4]. A complex nervous system, with central and peripheral systems, recapitulates the function of individual neurons: sensory neurons collect information from the environment, transmit this

* Address correspondence to this author at the Laboratory for Neuroscience, Department of Biological Sciences, University of Massachusetts Lowell, Lowell, MA 01854 USA; Tel: 978-934-2881: Fax: 978-934-3044

E-mail: Thomas_Shea@uml.edu information to inter-neurons where it is integrated, and a response is mediated by motor neuronal output (Fig. 1B). To consider the integration of nervous system function, the sensory neurons as a collective can be considered as analogous to dendrites of a single neuron, the interneurons analogous to the soma of that neuron, and the motor neurons as analogous to the axonal output of that neuron.

While we tend to regard ourselves as intellectual creatures, it is important to consider that our nervous system, including extremely well-developed cortical regions, shares overall homology with that of other animals [5] and remains critically dependent upon sensory input. Our interaction with our environment, including learning, logic, and formation of memories, is still filtered through the limbic system. It has been suggested that primitive emotions may underly the evolutionary development of consciousness and that aspects of consciousness are experienced by animals as well as humans [6 - 9]. "Neural Correlates of Consciousness" are considered to be the physical cortical regions that generate aspects of consciousness $[6,10]$. Moreover, consciousness can be defined as relatively simple responses to the environment, adaptation, and establishment of long-term memory (considered as "primary consciousness") versus self-awareness and complex 
language ("higher-order consciousness") [11]. Higher-order consciousness may be restricted to humans and/or primates [11], and primary consciousness is widely present among animals $[12,13]$. This line of reasoning is consistent with the wide-spread distribution of general neuronal features among animals [14]. Consciousness, especially higher-order consciousness, may be considered as the ultimate emergent property of the nervous system, and its emergence, like any neuronal response, can be distilled down to the integration of synaptic transmission.

The reductionist approach of monitoring neuronal interactions in culture provides a unique analysis of the nervous system development and function of the nervous system. Resultant ex vivo neuronal networks display multiple aspects of primary consciousness, including complex signaling regulated by the extent of synaptic connections, responsiveness to the environment including the appropriate direction of motor activity, and long-term potentiation (which is the cellular basis for learning and memory). We will examine these in detail in the following sections.

We discuss these topics both in the context of a review/overview as well as to serve as potential starting points for culturing of neurons on MEAs. Illustrations and text are drawn largely from our previous publications, with appropriate references in the text and figure legends. Readers are encouraged to review these prior publications for more detail.

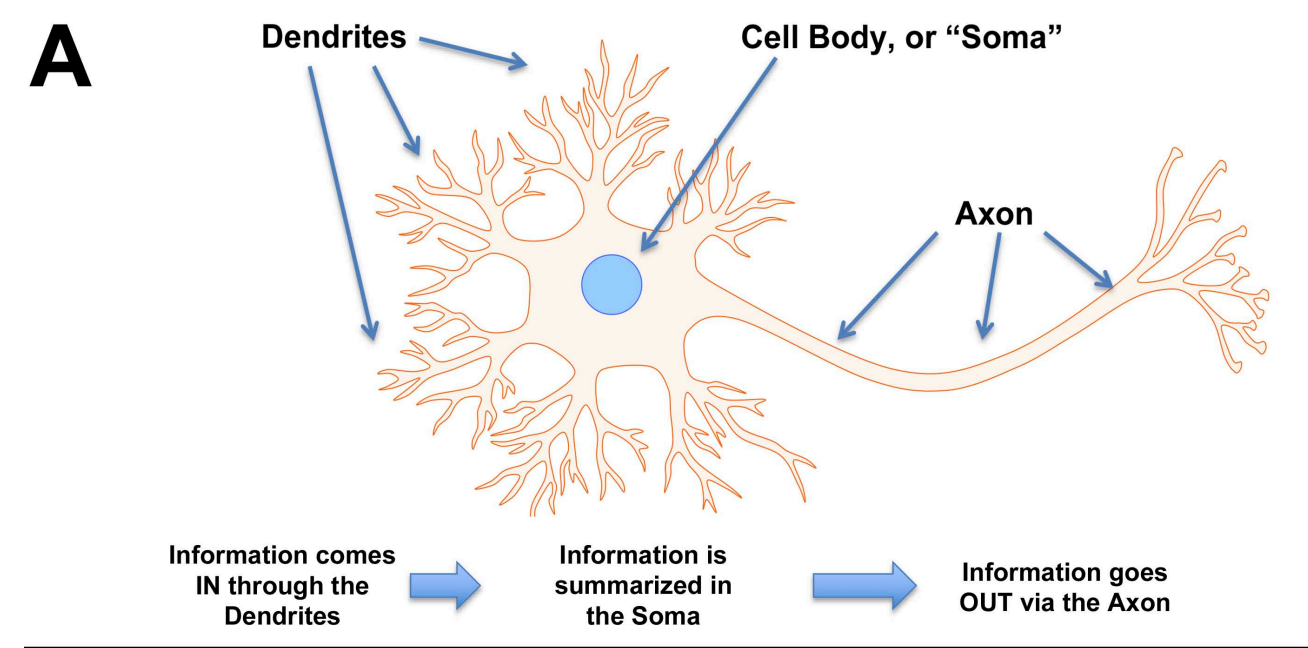

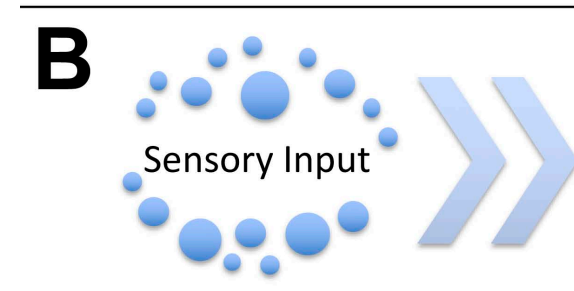

Touch, heat, pain seeing, hearing

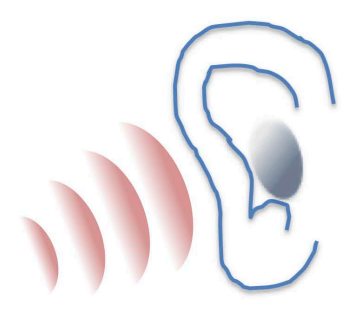

Peripheral Input

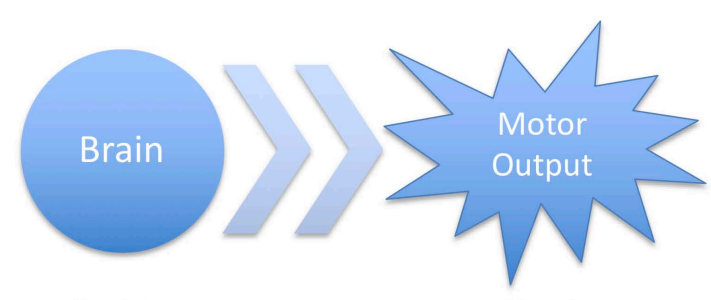

Decision

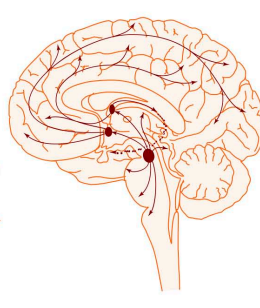

Central Processing
Muscle

Movement

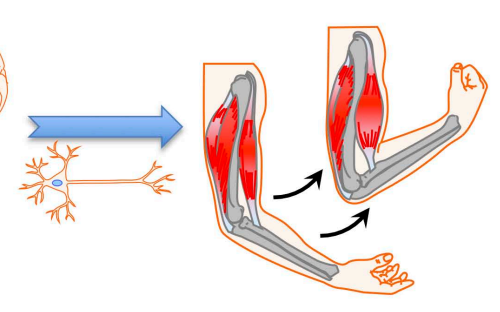

Peripheral Output

Fig. (1). Diagrammatic representation of collection, integration, and action in response to sensory input. Panel A presents an individual neuron, where dendrites collect sensory input, which is integrated at the cell body and summarized as a "fire/no fire" action via the axon and associated synapse. Panel B presents a complete, complex nervous system. Central and peripheral systems recapitulate the function of individual neurons: sensory neurons collect information from the environment, transmit this information to inter-neurons where it is integrated, and a response is mediated by motor neuronal output. 


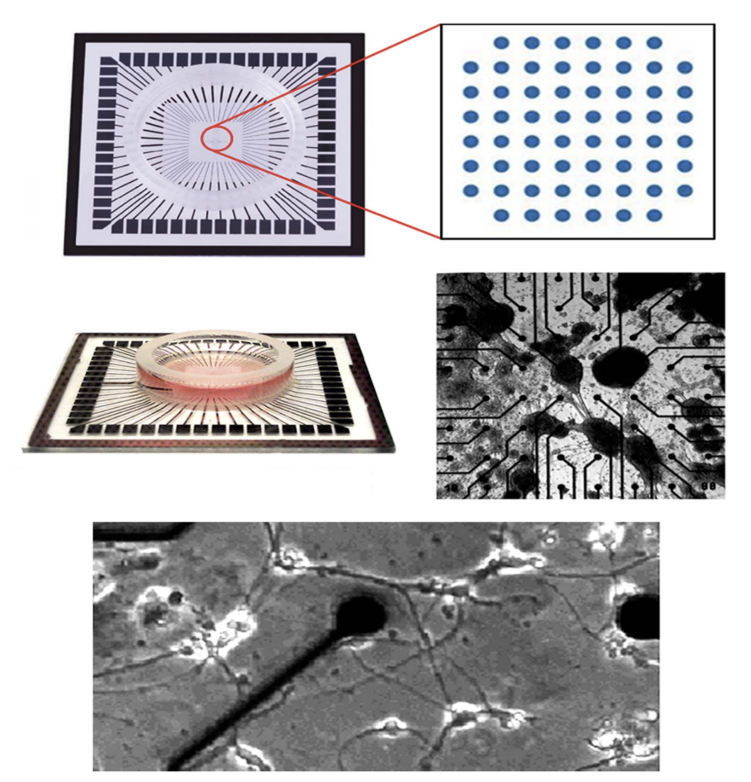

Fig. (2). Multi-electrode arrays (MEA). The image presents a diagram of MEAs (top row), a photograph of a MEA that more clearly depicts the culture dish situated above the electrode array (Left of middle row), and a micrograph of neuronal clusters within the MEA. The bottom panel presents a higher magnification image of neurons on a MEA; this image contains an electrode with its associated connecter and axons elaborated by neurons cultured on the MEA. MEAs are capable of recording the electrical activity of cultured neurons.

\section{GENERAL METHODS}

Establishing ex vivo neuronal networks is accomplished via the basic methods for culturing neurons, in either dissociated or slice versions, with the only difference being that electrodes are embedded in the culture chamber base. These methodologies are presented in detail in the primary articles e.g., [15 - 26]. Particular methodologies are summarized in each of the following sections.

Regarding eligibility criteria for studies reviewed herein, in accordance with Cochrane Community guidelines (https://community.cochrane.org/mecir-manual/standards-cond uct-new-cochrane-intervention-reviews-c1-c75/developingprotocol-review-c1-c23/setting-eligibility-criteria-includingstudies-review-c5-c13), we made every attempt to include all relevant references, resulting in 81 total references, and did not deliberately exclude any MEA studies.

\section{DEVELOPMENT OF SYNAPTICACTIVITY: MONITORING BY MULTI-ELECTRODE ARRAYS AND COMPUTER INTERFACE}

Microelectrode Arrays (MEA) consist of a series of electrodes, typically embedded within a petri dish, that provide the ability to record neuronal activity. MEAs have been used in many studies to stimulate individual and/or multiple neurons and record their responses [15 - 17]. MEAs allow direct delivery of multiple spatiotemporal patterns of stimulation, in a manner not achievable in situ [18 - 20].

Dissociated cultures of cortical cells grown on multielectrode Arrays (MEAs) have been used in a wide variety of studies of network physiology. These studies described fundamental properties of neuronal network activity patterns, plasticity, models of learning and epilepsy, and pharmacological testing [20 - 26].
Various MEAs are commercially available (Fig. 2). They can be used to analyze signaling during the establishment of networks as well as during network manipulation via stimulation or treatment with various agents [18 - 20, 22, 23].

\section{SPONTANEOUS DEVELOPMENT OF COMPLEX SIGNALING IN NEURONS}

In dense cultures, neurons are often aggregated (e.g., Fig 3A). Minor baseline fluctuations observed in the absence of Bone fide signals but in the presence of neurons were readily distinguished from the minor baseline fluctuations observed in their absence (Fig. 3B). Cessation and restoration of this activity following addition and withdrawal of synaptic blockers such as tetrodotoxin confirmed this activity in the presence of neurons to be synaptic (Fig. 3B). Spontaneous signals, which occurred as individual spikes at irregular intervals, were observed within one week following culturing. By contrast, at 20 days after plating, streams of continuous spikes and "bursts" (defined as clusters of signals in which deviated from baseline for at least $0.7 \mathrm{sec}$ ) [27] were regularly appeared (Fig. 3C). These streams predominated for the following 15 days. Bursts persisted throughout the time during which cultures were monitored [27]. Over time, the number of spikes decreased while bursts persisted, which eventually resulted in an overall prevalence of bursts within 1 month after plating [27]. Astroglia were routinely allowed to proliferate and typically obscured the neuronal network over time (Fig 3E and F). Long-term cultures ( $\geq 1$ month in culture), operationally defined as "mature" cultures, displayed spontaneous signals that radiate across substantial regions, recorded by the simultaneous appearance at multiple electrodes, yet absent from adjacent electrodes (Fig. 3G and $\mathbf{H}$ ) [28]. These findings indicate functional synaptic connectivity of neurons within cultures, resulting in a neuronal network. Networks remained functional for months [29]. We did not examine whether or not 
there was any selectivity among neurons for synaptogenesis. However, networks were generated by random deposition of neurons into a 2-dimensional surface, lacking the architectural restrictions and organizing aspects observed in situ [30]. Therefore, synaptogenesis in these ex vivo neuronal networks can be considered as occurring on a "nearest-neighbor" basis.

The central nervous system contains excitatory and inhibitory neurons, and its functionality is dependent upon their interaction. We examined whether or not the interaction of excitatory and inhibitory neurons was responsible for the development of mature spontaneous signaling patterns. To accomplish this, bicuculline, an antagonist of receptors for the inhibitory neurotransmitter gamma-aminobutyric acid (GABA), for $2 \mathrm{~h}$ [31] was added to neuronal networks. The addition of bicuculline increased the number and amplitude of signals. This indicated that inhibitory neurons were responsible for restricting overall spontaneous activity Fig. (4) [32, 33]. Notably, the overall loss of signals following the addition of this excitatory antagonist confirms that signals, including the increase following the addition of bicuculline, are derived from the excitatory neuronal synaptic activity.
The emergence of complex signaling (i.e., including bursts) was density-dependent. Sparse neuronal cultures did not convert to bursts but instead displayed continuous spikes, while dense cultures displayed predominantly bursts over time (Fig. 5) [32]. Similar results were obtained for signaling in thin and thick areas of the same culture. Since a small percentage of neurons were GABAergic, we considered previously that a resultant small number of inhibitory synaptic connections formed within thinner cultures and that this reduced number was insufficient to regulate excitatory activity. These results are consistent with these prior considerations. The $3>$ fold increase in signaling following bicuculline addition of bicuculline to established cultures [32], coupled with only $25 \%$ of total neurons displaying GABA, suggests that inhibitory neurons control more $>1$ excitatory neuron. These findings suggest that, on average, a single inhibitory neuron regulates the activity of 3 excitatory neurons. This is in line with prior demonstrations in a study that stated that inhibitory neurons control multiple excitatory neurons during development [34]. Multiple synaptic arrangements could mediate the ability of one inhibitory neuron to regulate the activity of multiple excitatory neurons (Fig. 6).
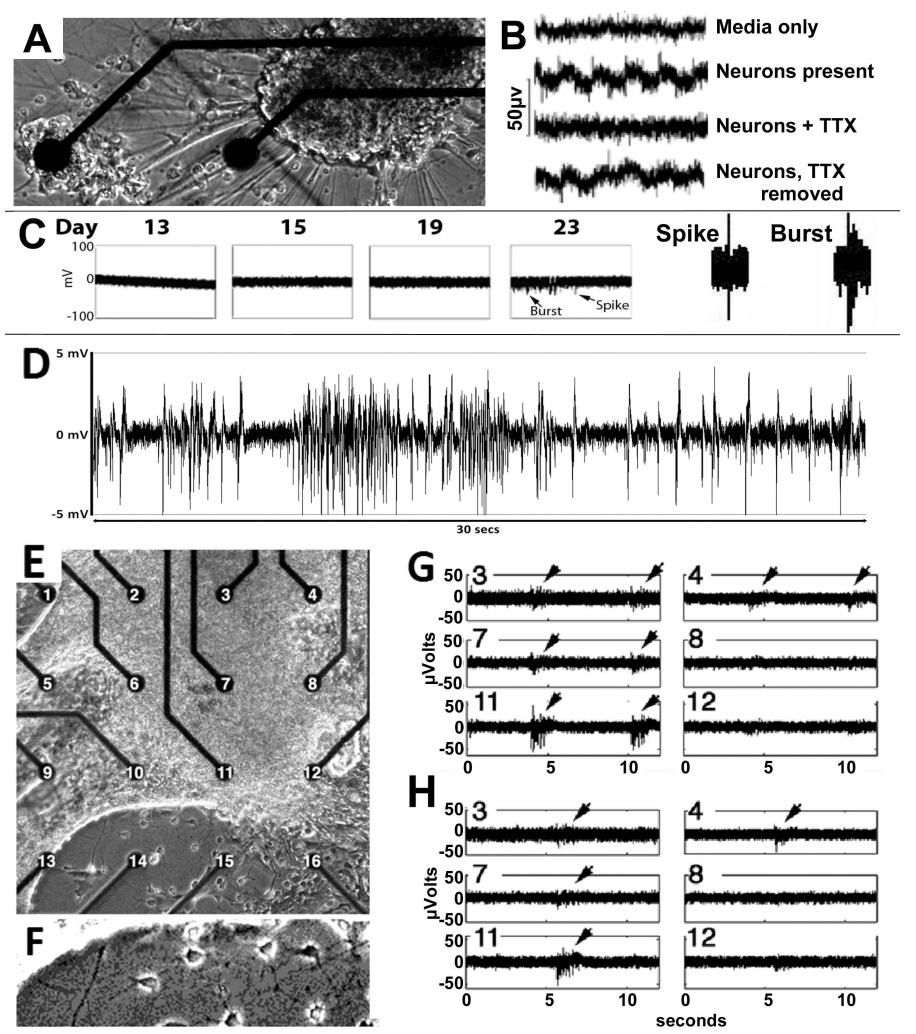

Fig. (3). Neurons establish a functional network ex vivo. Panel A presents a representative phase-contrast image of neurons approximately 1 month after plating on a MEA. A large aggregate with elaborating neurites is presented. Two electrodes and their connectors are presented. Panel B presents 1 -sec recordings of a mature culture. The first recording presents the minor baseline fluctuations observed with neurons present in the culture. The second presents signaling observed in the presence of neurons. Signaling was reversibly inhibited following the addition and removal of the synaptic blocker tetrodotoxin TTX. Panel C presents recordings over time, with respective days after plating listed above each panel. Representative spikes and bursts are indicated. A representative spike and a burst are also presented at higher magnification. Panel D presents a region of a mature culture; astroglial proliferation often obscures visualization of the neuronal network. Electrodes are arbitrarily numbered that highlight the variation among signals. Panel E presents the lower region of the image in D at higher magnification to allow visualization of neurites. Panels $\mathrm{G}$ and $\mathrm{H}$ present different $15 \mathrm{sec}$ recordings from the indicated electrodes of the network presented in panel D. Arrows indicate synchronous signals at some but not all electrodes, indicating the establishment of synaptic connections; portions from [28]. 

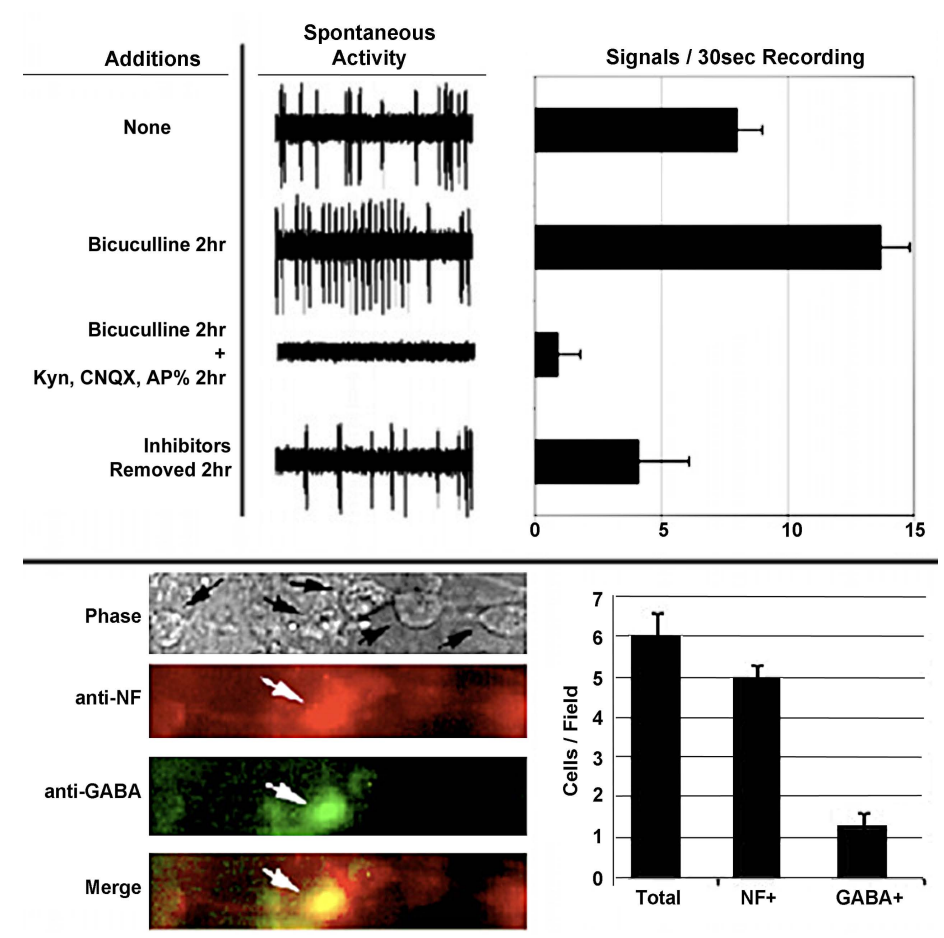

Fig (4). Inhibitory neurons restrict spontaneous signaling. Panel A presents representative 30 sec recordings of spontaneous signals obtained before and $2 \mathrm{hr}$ after bicuculline addition, $2 \mathrm{hr}$ after the excitatory inhibitor cocktail addition (with bicuculline still present), and $2 \mathrm{hr}$ after all agents were removed. Note the relative increase in signaling following the addition of bicuculline ( $p<0.05$ versus control ( $i . e$., no additions)). Note that all signals ceased following the addition of excitatory inhibitors $(p<0.05$ versus control and bicuculline alone). Note also that signaling resumed following removal of inhibitors $(p<0.05$ versus the presence of all inhibitors and statistically identical to control). Panel B presents micrographs of cultures subjected to double-immunofluorescent analyses for neurofilaments (anti-NF) using the monoclonal antibody SMI-32 and a polyclonal antibody directed against the GABA receptor (anti-GABA), followed by Texas Red- and fluorescein-conjugated secondary antibodies, respectively. All neurons (black arrows) contained NFs, but a subset contained GABA(white arrow). Quantification of total and GABA-positive neurons from three such cultures is displayed in the accompanying graph; from [32].
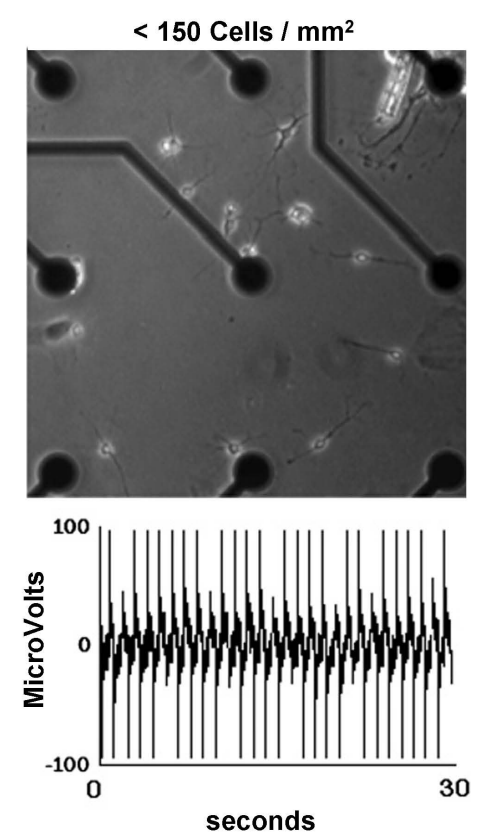
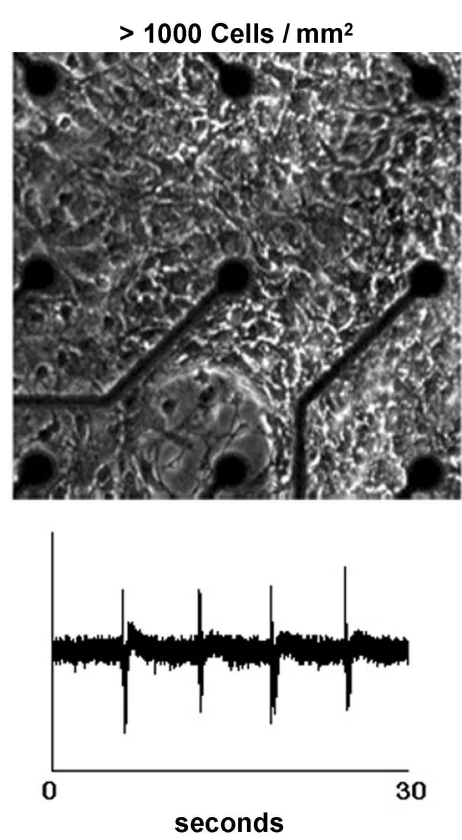

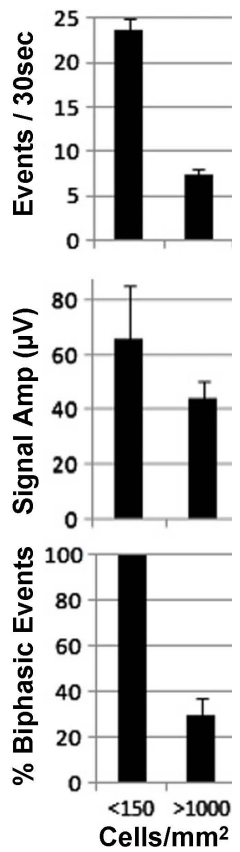

Fig (5). Culture density modulates signaling patterns. We compared the signaling patterns of networks with $<150$ cells per mm ${ }^{2}$ (“thin") as shown, with those with $>1000$ cells per $\mathrm{mm}^{2}$ ("thick"). Images depict representative thin and thick networks, spontaneous signals recorded from these networks, and quantification of the number, amplitude, and biphasic degree of these signals. Note that thin networks displayed more high-amplitude and biphasic signals than thick networks $(p<0.05)$; from [32]. 


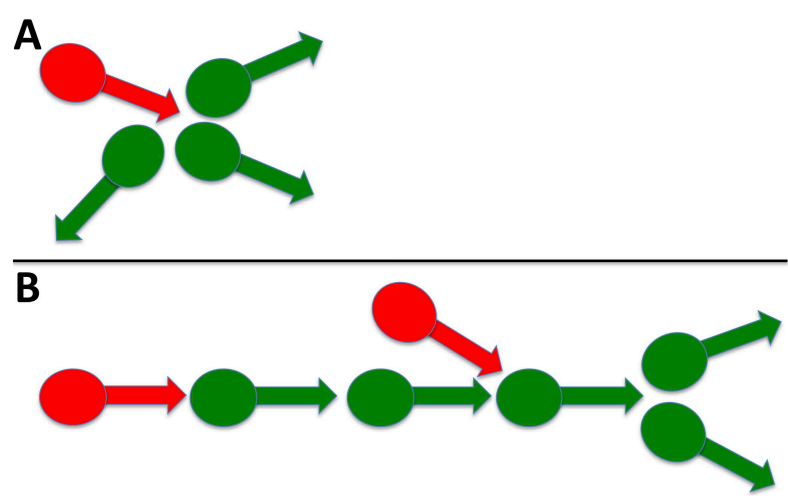

Fig. (6). Inhibitory neurons regulate multiple excitatory neurons. Panels present two possible arrangements by which inhibitory neurons could accomplish this. In panel A, a single inhibitory neuron (colored in red) directly synapses with 3 excitatory neurons (colored in green). In panel B, inhibitory neurons synapse with a single excitatory neuron that synapses with additional downstream excitatory neurons that could branch or merge. Additional synaptic arrangements are possible.

We routinely allowed astroglial proliferation in our ex vivo neuronal networks; we and others have demonstrated that factors released by glial cells increased complex signaling [28, $32,35]$.

\section{THE ROLE OF INHIBITORY NEURONS IN THE DEVELOPMENT, MATURATION, AND AGING OF THE NERVOUS SYSTEM IS RECAPITULATED IN EX VIVO NETWORKS}

A developmental delay in cortical inhibitory activity is observed in situ. Whether or not this eventual appearance of inhibitory activity is derived from excitatory neurons or by the transient excitatory activity of GABAergic neurons during development is unclear. We utilized the reductionist approach afforded by ex vivo neuronal networks to examine this possibility.

Between 20-30 days in culture, individual signals ("spikes") from $0.2-0.4 \mathrm{mV}$ in amplitude appeared, which was followed by high amplitude epileptiform activity. Bursts (defined as clusters of $\geq 3$ low-amplitude spikes within $0.7 \mathrm{sec}$ ) remained following the decline of epileptiform activity (Fig. 7). Notably, the bicuculline had no effect on the initial signaling but subsequently fostered continued epileptiform activity (Fig. 7). This suggested that GABAergic neurons were functioning as inhibitory neurons at this point [36].
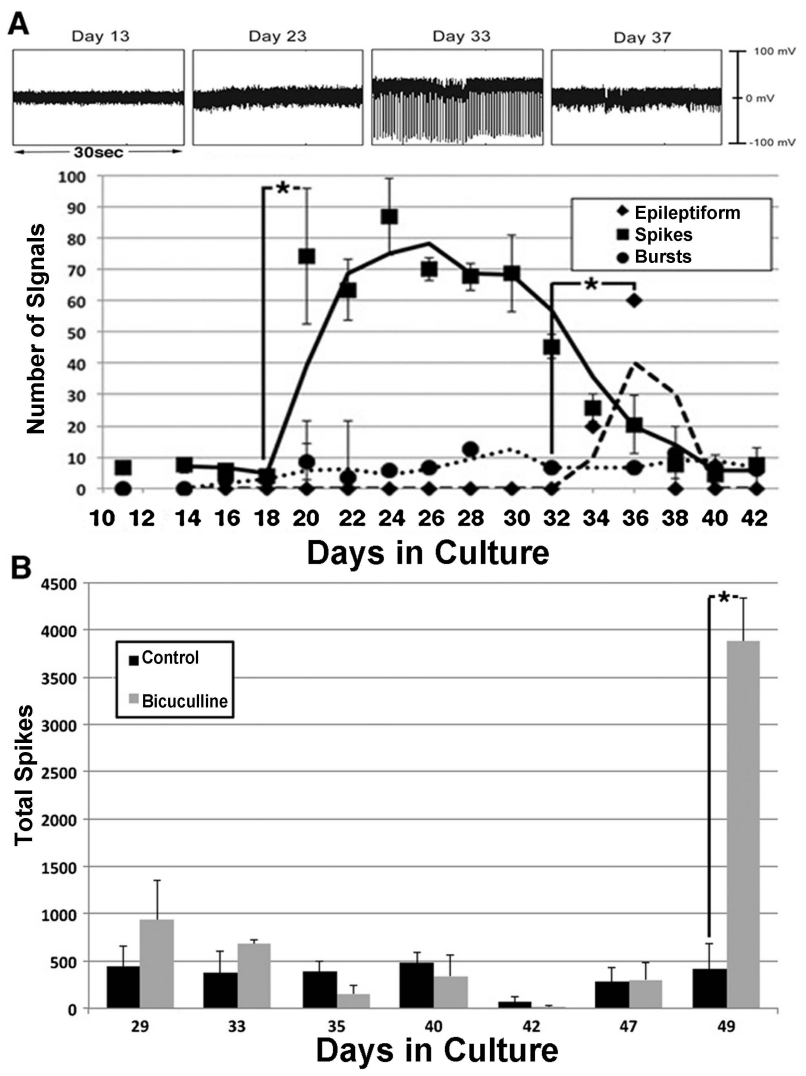

Fig. (7). Networks undergo a transient epileptiform phase: Panel A presents representative recordings of a network on the indicated day after plating. Signals were present by day 23. A transient "epileptiform" phase of very high amplitude ( $>0.5 \mathrm{mV})$ spikes was present at day 33 . The accompanying graph quantifies spikes, bursts, and epileptiform signals each day from 10 to 42 days after plating quantified from multiple networks (Zemianek et al., 2012b). Panel B quantifies total spikes of any amplitude in the presence and absence of bicuculline from day 29 to day 49 after plating. Bicuculline had no effect from day 29 to 47 and resulted in a statistically significant increase in spikes at day 49; from [36]. 
These results are consistent with the delayed formation of GABAergic synapses [37, 38], and with transient excitatory activity by newly-formed GABAergic synapses [39]. Additional studies have demonstrated an age-related decline in inhibitory neuronal activity $[31,40]$, which may contribute to the dysregulation of inhibitory activity and resultant aberrant excitatory activity observed in several age-related neurodegenerative conditions [40, 41].

This transient excitatory activity of GABAergic neurons also contributed to the level of total excitatory signaling required to trigger the developmental conversion of initially excitatory GABAergic neurons to inhibitory neurons [42]. Moreover, insufficient GABAergic excitatory activity actually inhibited this developmental transition. This leaves open the possibility that failure to sufficient GABAergic neurons to convert from excitatory to inhibitory could leave an individual at a threshold for seizures [42]. One interpretation of these latter findings is that epilepsy could arise not from excessive excitatory activity but rather from a lack of sufficient inhibitory activity. These latter findings underscore the ability of the reductionist approach of ex vivo neuronal networks to elucidate aspects of nervous system development and homeostasis that have remained difficult if not impossible to clarify in situ.

\section{EX VIVO NEURONAL NETWORKS DISPLAY COMPLEX RESPONSES TO STIMULI}

Rather than functioning in isolation, nervous systems from simple to complex benefit their host organism by receiving and processing information from the environment via sensory input. We and others took advantage of the ability to deliver electrical stimulation to networks via the MEA, which provides a model of sensory input.

A biphasic square wave signal utilizing the bath ground electrode as the distal grounding electrode was first applied Fig. (8A, left panel), which resulted in a 2.7 -fold increase in signals from the network [43]. We wondered whether or not stimulation with more complex signals would foster the network to display a more complex response. We first applied the same biphasic square wave 3 times in rapid sequence (Fig. 8A), which induced a 1.5 -fold increase in network signals versus those elicited by a single stimulation.

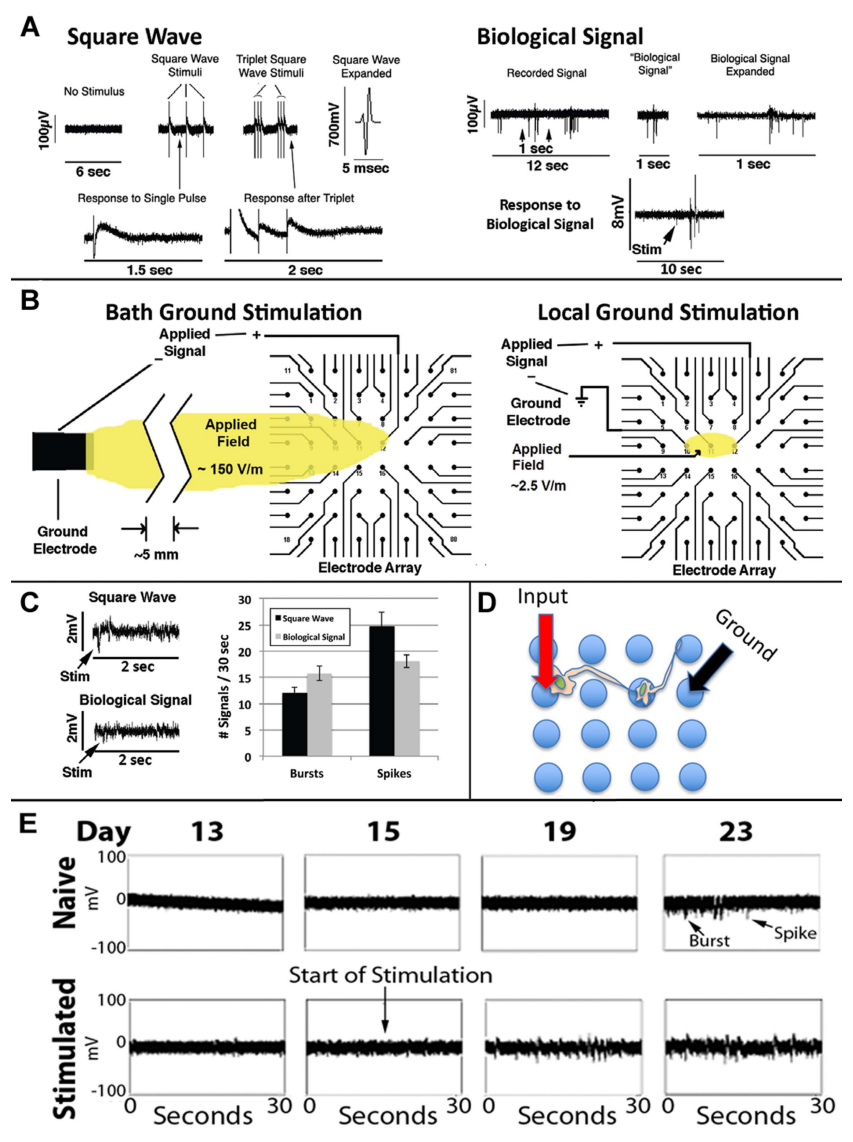

Fig. (8). Panel A: Representative signals from an unstimulated mature network ("No stimulus"), single and triple biphasic square wave signals, the Biological Signal, and network responses to each. Panel B: Electric field generated using the bath ground electrode and that following localized stimulation. Panel C: Network responses following localized stimulation Panel D: Diagram of localized stimulation depicting how a neuron situated on the stimulated electrode could generate a downstream response of a second, synaptically connected neuron, which could be detected at an electrode in contact with the downstream neuron. Panel E: Network response following a single stimulation with the Biological Signal on day 15 after plating, which hastened the appearance of both spikes and bursts. Modified from [27]. 
Since networks were not receiving any external stimulation spontaneously developed complex signaling patterns, including conversion of initially excitatory neurons to inhibitory neurons $[27,43]$, we considered that an actual neuronal signal might invoke even more complex responses than biphasic square waves. We, therefore, extracted a $1 \mathrm{sec}$ portion from a recorded spontaneous signal from a mature network (defined as the "Biological Signal"; Fig. 8A, right panel). We amplified the Biological Signal to $1 \mathrm{mV}$ in amplitude, which invoked a response at least as robust as the above square wave signals that themselves were substantially larger in amplitude $(700 \mathrm{mV}$; Fig. 8A).

We then developed a method for localized stimulation of portions of the network, using 3 of the MEA electrodes (Fig. 8B).

We then compared localized stimulation of networks with the square wave and Biological Signal. The Biological Signal induced more bursts versus individual signals $(p<0.03$; Student's $t$-test; Fig. 8C). We examined the consequences of early, localized stimulation with the Biological Signal on network development. Stimulation at day 15 hastened the appearance of both spikes and bursts (Fig. 8E) as well as the transient developmental epileptiform phase [36].

\section{EX VIVO NEURONAL NETWORKS CAN UNDERGO LONG-TERM POTENTIATION}

The ability of the nervous system to learn and establish memory evolves perhaps more than once because of survival pressures for increasingly complex organisms. Synaptic alteration, often referred to as long-term potentiation, is considered to be the basis for learning [44 - 50].

We previously demonstrated that network stimulation during development fostered an earlier appearance of mature signaling patterns (Fig. 8) [27, 36, 43]. We undertook to examine whether or not more extensive stimulation could invoke a long-term alteration in signals elaborated by ex vivo neuronal networks.

Mature networks (i.e., >30 days after plating) were repetitively stimulated for $5 \mathrm{~min}$, twice daily at $6 \mathrm{hr}$ intervals for 5 days in the presence and absence of bicuculline.

Unstimulated networks displayed a mixture of spikes and bursts (Fig. 9), with bursts accounting for approximately 35\% of the total signals. No change was observed over 5 days in the absence of stimulation, but stimulation for 5 days increased total signals and bursts (Fig. 9). Bicuculline attenuated these increases. Increased signaling following stimulation indicates that mature networks can undergo synaptic plasticity, while prevention by bicuculline confirms a role for inhibitory neurons in this plasticity, consistent with findings in situ [52]. For reviews, see other research works as well [53 - 56].

\section{MODELING OF MOTOR OUTPUT IN RESPONSE TO SENSORY INPUT IN EX VIVO NEURONAL NETWORKS}

Since our networks responded to stimulation as a model of sensory input as described above, we designed a system that allowed this network to generate responsive output in terms of motor activity. The ex vivo network in this system functioned as interneurons - that is, essential as a central nervous system generating a motor response to sensory input.

The software interface can be obtained at https:/github .com/ab3nd/NeuronRobotInterface.The ROS module ("img slicer") (available at https://github.com/ ab3nd/NeuronRobot Interface/tree/master/catkin_ws/src/img_slicer/src) con-verted recorded video into HSV (Hue, Saturation, Value), and separated signals into hue, saturation, and value planes. The hue image was thresholded, and all pixels of the desired hue (red in this example) were converted to white, and all others converted to black (Fig. 10).

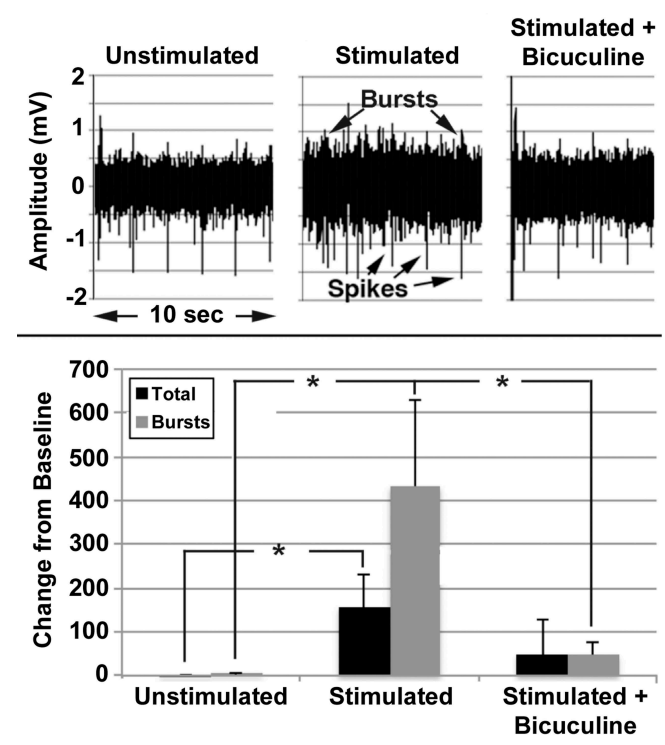

Fig. (9). Representative signal profiles following stimulation \pm bicuculine for 5 days along with that of unstimulated networks. An asterisk indicates $\mathrm{p}<0.05$ ), adapted from [43]. 


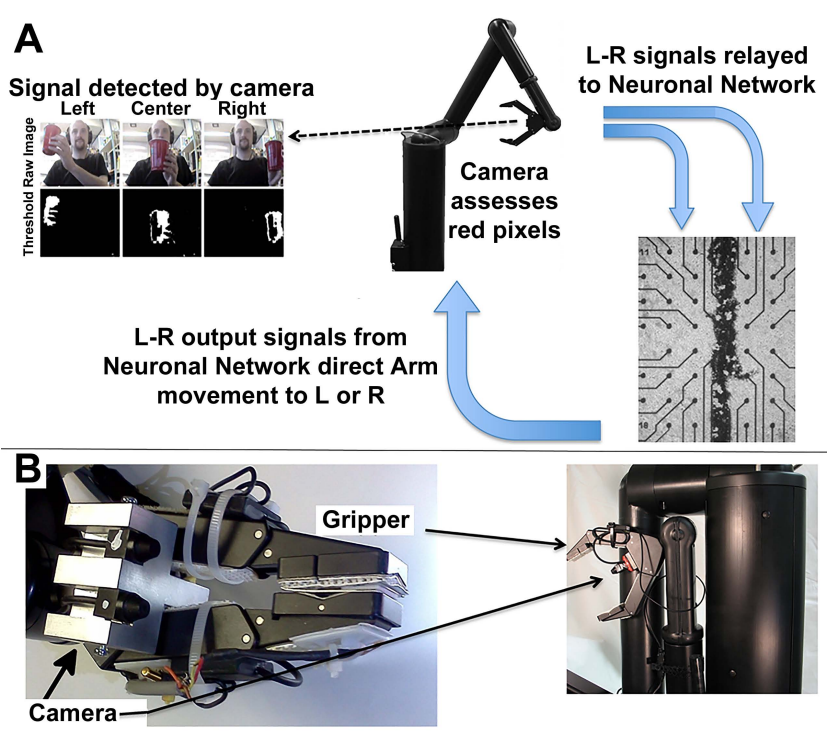

Fig. (10). Panel A: Flow of information through this system. The camera detects the target and signals the network, which in turn signals the arm to track the target. Panel B: The digital camera is mounted above the grasping hand of the Manus robot arm.

The field to be observed was separated into 5 segments while pixels summed and compared among these segments. If the majority of pixels were in the rightmost segments, the network was stimulated on its right side by the conversion of pixels into Biological Signals. This fostered increased synaptic signaling of the right portion of the network (Fig. 10). Resultant signals were converted to motion command for the Manus robotic arm by the ROS module, "act vector" (https:/github.com/ab3nd/NeuronRobotInterface/tree/master/c atkin_ws/src/activation_vector/src) [57]. The requirement for software-mediated translation of pixels into digitized synaptic signals, followed by the translation of synaptic signals into motion commands to direct the Manus arm remain consistent with physiological conversion of photons into synaptic signals and subsequent conversion of synaptic signals to direct actin and myosin interactions that mediate muscle motility.

Network activity in response to camera input and target tracking is shown in Fig. (11).

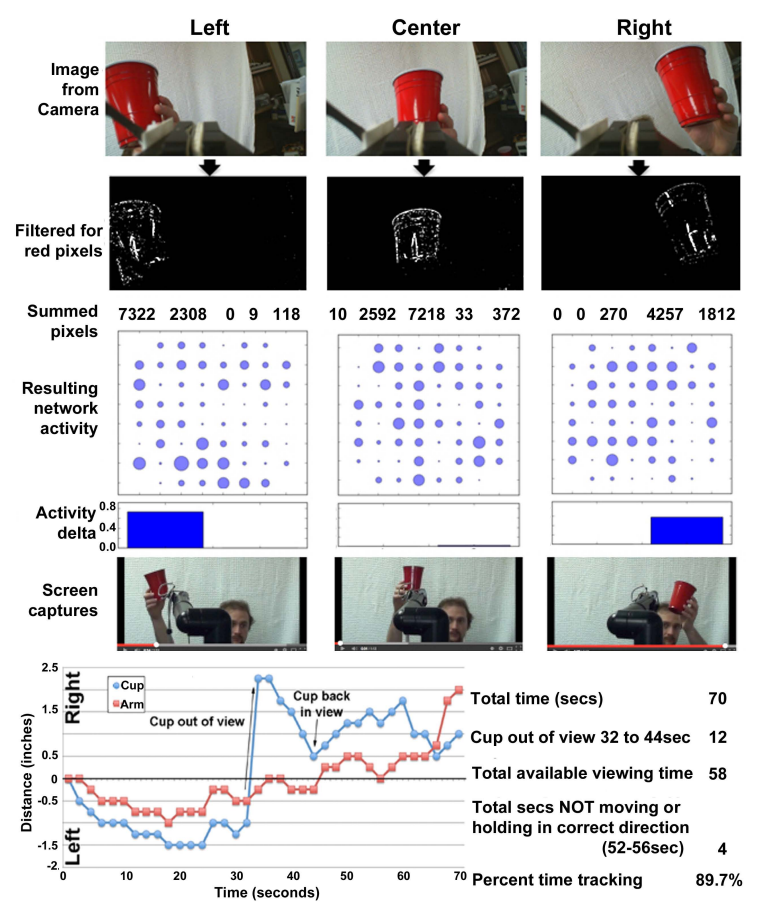

Fig. (11). Panels present the raw target image, the thresholded image, pixel distribution ("Summed pixels"), Resulting network activity," total activity, the difference across the field ("Activity delta") and representative screen that demonstrate tracking. The accompanying graph presents a representative tracking sequence. Analysis of this particular tracking sequence is presented to the right of the graph. 
The robot arm accurately tracked the target (Fig. 11).

Previous studies have utilized similar ex vivo networks to operate a small model vehicle $[58,59]$.

\section{RUDIMENTARY ASPECTS OF CONSCIOUSNESS ARE DISPLAYED IN EX VIVO NEURONAL NETWORKS}

After decades of largely philosophical consideration, consciousness has more recently been studied as a physiological activity [1, 4, 60 - 65]. "Neural Correlates of Consciousness" is considered to be the physical cortical regions that generate aspects of consciousness $[6,10]$. Notably, however, the development of the neocortex preceded humans and is present, albeit less well developed, in reptiles and mammals [5]. Aspects of consciousness are also observed in birds [6]. While more complex nervous systems are associated with more complex behaviors, it has been noted that behavioral complexity does not precisely correlate with nervous system complexity $[60,66]$. If consciousness is an emergent property of cortical activity, it is reasonable to consider that animals display some aspects of consciousness. In this regard, it has been suggested that primitive emotions may underly the evolutionary development of consciousness and that aspects of consciousness are experienced by animals as well as humans [6 - 9].

Along with this, consciousness can be divided into primary consciousness (which encompasses relatively simple responses to the environment, adaptation, and long-term memory) and higher-order consciousness, which allows future planning, selfawareness, and complex language [11]. While higher-order consciousness may be restricted to humans and perhaps other primates [11], it is considered that the primary aspects of consciousness are widely present among animals [12, 13], which is consistent with the wide-spread distribution of general neuronal features among animals [14]. The neural activity underlying primary consciousness must still be present in animals with higher-order consciousness [11]. In this regard, ex vivo neuronal networks indicate multiple aspects of primary consciousness, including complex signaling regulated by the extent of synaptic connections, responsiveness to the environment including the appropriate direction of motor activity, and long-term potentiation (which is the cellular basis for learning and memory).

Loss of higher-order consciousness is directly linked to perturbation of neuronal interactions, supporting the notion that consciousness is an emergent property of neuronal interaction $[6,67]$. Since the loss of organized synaptic activity is associated with disruption of consciousness, it is conceivable that the timing or synchronization of neural activity, rather than simply the overall level of spiking, might correlate with or mediate awareness [68]. Which region give rise to consciousness is not entirely clear and may involve the upper brain stem in addition to, and by interaction with, cerebral cortical regions, suggesting that relatively simple signaling contributes to consciousness [69, 70].In neurodegenerative conditions such as Alzheimer's disease, following traumatic injury or administration of anesthesia, consciousness can be lost, yet portions of the cerebral cortex may still remain active with individuals persisting in a so-called "vegetative state" [71]. Notably, the same agents that perturb neuronal signaling leading to impaired consciousness in situ also perturbed neuronal signal in ex vivo neuronal networks, such as the betaamyloid peptide that accumulates in Alzheimer's disease [72 74], the neurotoxin tetrodotoxin, the GABAergic antagonist bicuculline, various excitatory antagonists $[32,33]$, and excess extracellular glutamate [75]. This is consistent with in situ studies suggesting the contribution of relatively simple neuronal interaction to consciousness $[11,76]$.

\section{DISCUSSION}

The nervous system allows an organism to receive and react to information from the environment. The nature and range of potential reactions increases in complexity with increasingly developed nervous systems. No matter how complex and diverse these reactions may be, they are ultimately derived from simple synaptic interaction of excitatory and inhibitory neurons as modulated by the architecture of the organism's nervous system. This is underscored by studies with ex vivo neuronal networks. Our studies and those of other laboratories demonstrate that ex vivo neuronal networks can model multiple aspects of the nervous system, including development [42], maturation [36], longterm potentiation [43], environmental interaction [43, 57], aging [31] and ultimate neurodegeneration [72 - 75]. While the "default" interpretation of neuronal behavior is perhaps to consider stimulation as evoking response, our studies underscore the additional requirement of inhibitory neuronal activity for the achievement of both the development as well as maintenance of complex "responsive" actions of the nervous system. The reductionist approach of simple ex vivo neuronal networks may continue to provide insight into the complex functionality of nervous systems in situ.

Results obtained with ex vivo neuronal networks in several cases are paralleled with the results generated using computergenerated neural networks. This included our demonstration of the role of inhibitory neurons in complex signaling, development of the nervous system, and in an embodiment of networks with visual input and motor output [32, 33, 77]. This underscores the potential usefulness of ex vivo neuronal networks to probe the physiological basis of phenomena observed with computer-based neural networks.

\section{GETTING STARTED WITH MEAS AND Ex Vivo NEURONAL NETWORKS}

Multiple complete commercial MEA systems are available on a number of software and hardware platforms. Commercial systems, unfortunately, require a level of financial investment that can be prohibitive for laboratories that require extramural support since preliminary data justifying such support cannot be obtained in the absence of a computer-neuronal network interface. Our initial system used a custom 16-pin interface; details and images are presented in another study [28]. Despite the requirement of multiple relocations to access all channels, this simple system was capable of recording and stimulation [27]. We subsequently incorporated a 64-channel interface and developed a custom software program "Raptor" that provides a simplified user interface and can export to Excel (Fig. 12); this 


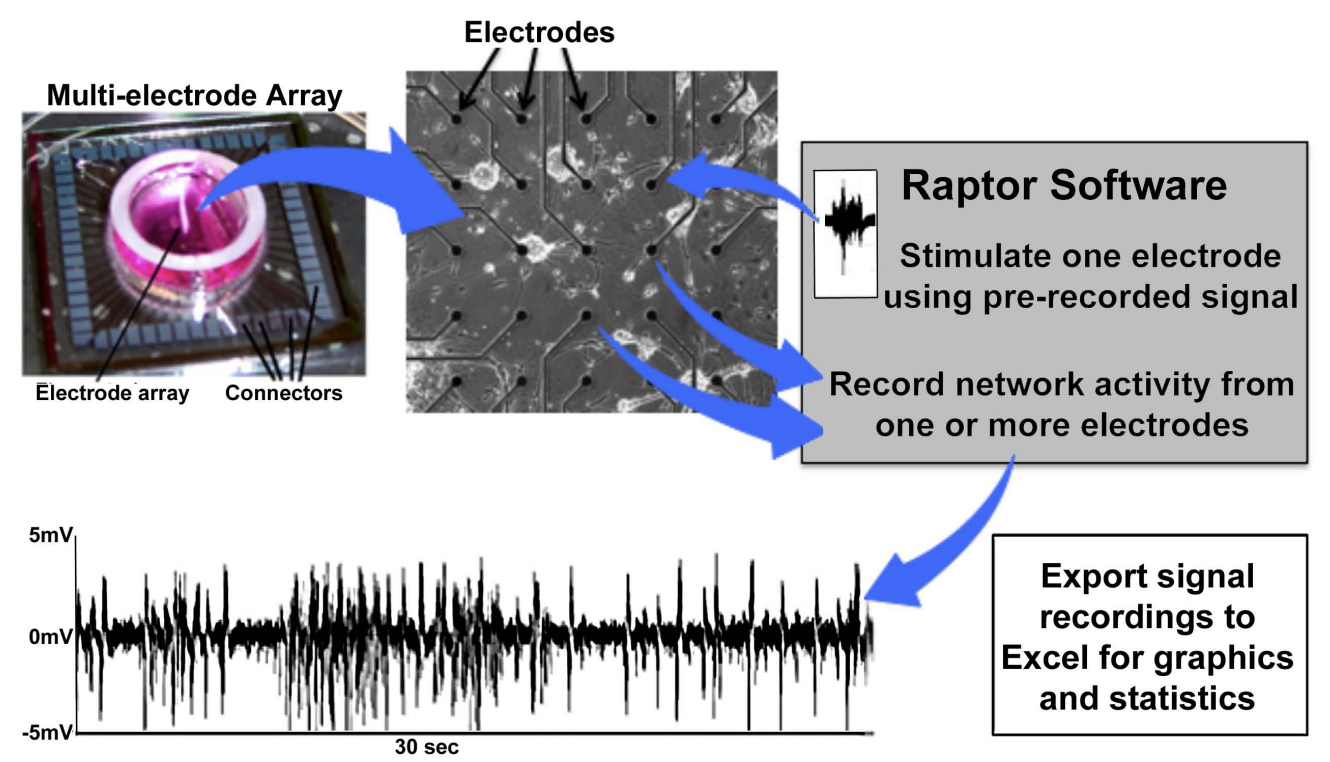

Fig. (12). Simplified Flow Chart for Recording with Raptor: Generation of neuronal networks on MEAs has been described in multiple articles, including those from our laboratory. Notably, however, the best starting point is an excellent article that includes video presentations of how to generate and utilize ex vivo neuronal networks [78].

program, utilized for most of the studies presented herein, is freely available to all investigators (contact the author or visit https:/github.com/mtgjbird/Raptor). Raptor requires LabView (National Instruments) run-time engine 2013 SP1 or later (http://www.ni.com/download/labview-run-time-engine-2013-s p1/4540/en/). Other appropriate software are available in different studies [78, 79]. Another low-cost MEA system that also utilizes LabView has been described previously [80].

\section{CONCLUSION}

Ex vivo neuronal networks established on MEAs provide reductionist models that allow analyses of normal development as well as for perturbations that accompany aging and other neuropathological conditions and for the screening of potentially therapeutic compounds [81]. Imbalance of excitatory and inhibitory neuronal activity, readily achievable in ex vivo networks, may be especially useful in studies of neurological disorders involving GABAergic dysregulation of, including epilepsy, schizophrenia, age-related neuropathic pain, Parkinson's disease, and Alzheimer's disease [40, 41]. Further study may elucidate other roles of inhibitory neuronal activity related to the normal and abnormal development and maintenance of the nervous system.

\section{CONSENT FOR PUBLICATION}

Not applicable.

\section{FUNDING}

This study has been sponsored by US Army Grants W911NF0810222, W911NF1010227, and W911NF1510206.

\section{CONFLICT OF INTEREST}

The authors declare no conflict of interest, financial or otherwise.

\section{ACKNOWLEDGEMENTS}

Figures and portions of the text presented in this review were taken from original publications sponsored by US Army Grants W911NF0810222, W911NF1010227, and W911N F1510206 to the author. The author is grateful for the continued advice of Dr. Elmar Schmeisser.

We thank the journals in which some of these figures appeared previously for granting permission to utilize figures, or portions of them, in this review. Each such figure has a reference in its legend to the appropriate source.

\section{REFERENCES}

[1] Storm JF, Boly M, Casali AG, et al. Consciousness regained: Disentangling mechanisms, brain systems, and behavioral responses. J Neurosci 2017; 37(45): 10882-93.

[http://dx.doi.org/10.1523/JNEUROSCI.1838-17.2017] [PMID: 29118218]

[2] Kinnamon JC, Westfall JA. Types of neurons and synaptic connections at hypostome-tentacle junctions in Hydra. J Morphol 1982; 173(1): 119-28.

[http://dx.doi.org/10.1002/jmor.1051730110] [PMID: 7108966]

[3] Westfall JA, Kinnamon JC. A second sensory motor interneuron with neurosecretory granules in Hydra. J Neurocytol 1978; 7(3): 365-79. [http://dx.doi.org/10.1007/BF01176999] [PMID: 207827]

[4] Arendt D, Tosches MA, Marlow H. From nerve net to nerve ring, nerve cord and brain evolution of the nervous system. Nat Rev Neurosci 2016; 17(1): 61-72.

[http://dx.doi.org/10.1038/nrn.2015.15] [PMID: 26675821]

[5] Cloninger CR. Evolution of human brain functions: The functional structure of human consciousness. Aust NZJ Psychiatry 2009; 43(11): 994-1006.

[http://dx.doi.org/10.1080/00048670903270506] [PMID: 20001395]

[6] Butler AB. Evolution of brains, cognition, and consciousness. Brain Res Bull 2008; 75(2-4): 442-9.

[http://dx.doi.org/10.1016/j.brainresbull.2007.10.017]

[PMID: 18331913]

[7] Denton DA, McKinley MJ, Farrell M, Egan GF. The role of primordial emotions in the evolutionary origin of consciousness. Conscious Cogn 2009; 18(2): 500-14.

[http://dx.doi.org/10.1016/j.concog.2008.06.009] [PMID: 18701321]

[8] Panksepp J. Affective consciousness: Core emotional feelings in 
animals and humans. Conscious Cogn 2005; 14(1): 30-80. [http://dx.doi.org/10.1016/j.concog.2004.10.004] [PMID: 15766890]

[9] Panksepp J. The basic emotional circuits of mammalian brains: Do animals have affective lives? Neurosci Biobehav Rev 2011; 35(9): 1791-804.

[http://dx.doi.org/10.1016/j.neubiorev.2011.08.003] [PMID: 21872619]

[10] Crick F, Koch C. Consciousness and neuroscience. Cereb Cortex 1998; 8(2): 97-107.

[http://dx.doi.org/10.1093/cercor/8.2.97] [PMID: 9542889]

[11] Edelman GM. Naturalizing consciousness: A theoretical framework. Proc Natl Acad Sci USA 2003; 100(9): 5520-4

[http://dx.doi.org/10.1073/pnas.0931349100] [PMID: 12702758]

[12] Fabbro F, Aglioti SM, Bergamasco M, Clarici A, Panksepp J. Evolutionary aspects of self- and world consciousness in vertebrates. Front Hum Neurosci 2015.

[http://dx.doi.org/10.3389/fnhum.2015.00157]

[13] Mather JA. Cephalopod consciousness: behavioural evidence. Conscious Cogn 2008; 17(1): 37-48.

[http://dx.doi.org/10.1016/j.concog.2006.11.006] [PMID: 17240163]

[14] Feinberg TE, Mallatt J. The nature of primary consciousness. A new synthesis. Conscious Cogn 2016; 43: 113-27.

[http://dx.doi.org/10.1016/j.concog.2016.05.009] [PMID: 27262691]

[15] Desai SA, Rolston JD, McCracken CE, Potter SM, Gross RE. Asynchronous distributed multielectrode microstimulation reduces seizures in the dorsal tetanus toxin model of temporal lobe epilepsy. Brain Stimul 2016; 9(1): 86-100. [http://dx.doi.org/10.1016/j.brs.2015.08.008] [PMID: 26607483]

[16] Pais-Vieira M, Lebedev M, Kunicki C, Wang J, Nicolelis MAL. A brain-to-brain interface for real-time sharing of sensorimotor information. Sci Rep 2013; 3: 1319.

[http://dx.doi.org/10.1038/srep01319] [PMID: 23448946]

[17] O'Doherty JE, Lebedev MA, Hanson TL, Fitzsimmons NA, Nicolelis MAL. A brain-machine interface instructed by direct intracortical microstimulation. Front Integr Nuerosci 2009; 3(3): 20.

[http://dx.doi.org/10.3389/neuro.07.020.2009] [PMID: 19750199]

[18] Wagenaar DA, Pine J, Potter SM. Effective parameters for stimulation of dissociated cultures using multi-electrode arrays. J Neurosci Methods 2004; 138(1-2): 27-37.

[http://dx.doi.org/10.1016/j.jneumeth.2004.03.005] [PMID: 15325108]

[19] Wagenaar DA, Potter SM. A versatile all-channel stimulator for electrode arrays, with real-time control. J Neural Eng 2004; 1(1): 39-45.

[http://dx.doi.org/10.1088/1741-2560/1/1/006] [PMID: 15876621]

[20] Wagenaar DA, Madhavan R, Pine J, Potter SM. Controlling bursting in cortical cultures with closed-loop multi-electrode stimulation. J Neurosci 2005; 25(3): 680-8.

[http://dx.doi.org/10.1523/JNEUROSCI.4209-04.2005] [PMID: 15659605]

[21] Beggs J M, Plenz D. Neuronal Avalanches in Neocortical Circuits. The Journal of Neuroscience 2003; 23 : 11167-77. [http://dx.doi.org/10.1523/JNEUROSCI.23-35-11167.2003]

[22] Jimbo Y, Robinson HP, Kawana A. Strengthening of synchronized activity by tetanic stimulation in cortical cultures: Application of planar electrode arrays. IEEE Trans Biomed Eng 1998; 45(11): 1297-304.

[http://dx.doi.org/10.1109/10.725326] [PMID: 9805828]

[23] Jimbo Y, Tateno T, Robinson HP. Simultaneous induction of pathwayspecific potentiation and depression in networks of cortical neurons. Biophys J 1999; 76(2): 670-8.

[http://dx.doi.org/10.1016/S0006-3495(99)77234-6] [PMID: 9929472]

[24] Maeda M, Ampo K, Kiryu-Seo S, et al. The p53-independent nuclear translocation of cyclin G1 in degenerating neurons by ischemic and traumatic insults. Exp Neurol 2005; 193(2): 350-60.

[http://dx.doi.org/10.1016/j.expneurol.2005.01.018] [PMID: 15869937]

[25] Shahaf G, Marom S. Learning in networks of cortical neurons. J Neurosci 2001; 21(22): 8782-8.

[http://dx.doi.org/10.1523/JNEUROSCI.21-22-08782.2001] [PMID: 11698590]

[26] Morefield SI, Keefer EW, Chapman KD, Gross GW. Drug evaluations using neuronal networks cultured on microelectrode arrays. Biosens Bioelectron 2000; 15(7-8): 383-96

[http://dx.doi.org/10.1016/S0956-5663(00)00095-6] [PMID: 11219752]

[27] Zemianek JM, Serra M, Guaraldi M, Shea TB. Stimulation with a lowamplitude, digitized synaptic signal to invoke robust activity within neuronal networks on multielectrode arrays. Biotechniques 2012; 52(3): 177-82.

[http://dx.doi.org/10.2144/000113823] [PMID: 22401551]

[28] Serra M, Dubey M, Chan A, Gilman V, Shea TB. A low-cost data acquisition interface for multi-electrode array culture systems. Biotechniques 2008; 45: 451-, 453-454, 456

[http://dx.doi.org/10.2144/000112911] [PMID: 18855772]

[29] Potter SM, DeMarse TB. A new approach to neural cell culture for long-term studies. J Neurosci Methods 2001; 110(1-2): 17-24. [http://dx.doi.org/10.1016/S0165-0270(01)00412-5] [PMID: 11564520]

[30] Kiecker C, Lumsden A. The role of organizers in patterning the nervous system. Annu Rev Neurosci 2012; 35: 347-67. [http://dx.doi.org/10.1146/annurev-neuro-062111-150543] [PMID: 22462542]

[31] Li X, Zhou W, Zeng S, Liu M, Luo Q. Long-term recording on multielectrode array reveals degraded inhibitory connection in neuronal network development. Biosens Bioelectron 2007; 22(7): 1538-43. [http://dx.doi.org/10.1016/j.bios.2006.05.030] [PMID: 16829068]

[32] Serra M, Guaraldi $M$, Shea TB. Inhibitory neurons modulate spontaneous signaling in cultured cortical neurons: Density-dependent regulation of excitatory neuronal signaling. Phys Biol 2010; 7(2)026009

[http://dx.doi.org/10.1088/1478-3975/7/2/026009] [PMID: 20526031]

[33] Zemianek JM, Lee S, Guaraldi M, Shea TB, Shea TB. Critical role for inhibitory neurons in modulation of synaptic signaling in ex vivo neuronal networks. Int J Dev Neurosci 2013; 31(5): 308-10.

[http://dx.doi.org/10.1016/j.ijdevneu.2013.03.011] [PMID: 23563174]

[34] Sahara S, Yanagawa Y, O'Leary DD, Stevens CF. The fraction of cortical GABAergic neurons is constant from near the start of cortical neurogenesis to adulthood. J Neurosci 2012; 32(14): 4755-61.

[http://dx.doi.org/10.1523/JNEUROSCI.6412-11.2012] [PMID: 22492031]

[35] Enright HA, Lam D, Sebastian A, et al. Functional and transcriptional characterization of complex neuronal co-cultures. Sci Rep 2020; 10(1): 11007.

[http://dx.doi.org/10.1038/s41598-020-67691-2] [PMID: 32620908]

[36] Zemianek JM, Shultz AM, Lee S, Guaraldi M, Yanco HA, Shea TB. Transient epileptiform signaling during neuronal network development: Regulation by external stimulation and bimodal GABAergic activity. Int J Dev Neurosci 2013; 31(2): 131-7. [http://dx.doi.org/10.1016/j.ijdevneu.2012.11.003] [PMID: 23220177]

[37] Briggs SW, Galanopoulou AS. Altered GABA signaling in early life epilepsies. Neural Plast 2011; 2011527605

[http://dx.doi.org/10.1155/2011/527605] [PMID: 21826277]

[38] Sutor B, Luhmann HJ. Development of excitatory and inhibitory postsynaptic potentials in the rat neocortex. Perspect Dev Neurobiol 1995; 2(4): 409-19. [PMID: 7757410]

[39] Ben-Ari Y. Excitatory actions of gaba during development: The nature of the nurture. Nat Rev Neurosci 2002; 3(9): 728-39. [http://dx.doi.org/10.1038/nrn920] [PMID: 12209121]

[40] Shetty AK, Bates A. Potential of GABA-ergic cell therapy for schizophrenia, neuropathic pain, and Alzheimer's and Parkinson's diseases. Brain Res 2016; 1638(Pt A): 74-87.

[http://dx.doi.org/10.1016/j.brainres.2015.09.019] [PMID: 26423935]

[41] Zhang CQ, McMahon B, Dong H, et al. Molecular basis for and chemogenetic modulation of comorbidities in GABRG2-deficient epilepsies. Epilepsia 2019; 60(6): 1137-49. [PMID: 31087664]

[42] Therrien M, Vohnoutka R, Boumil E, Guaraldi M, Lee S, Shea TB. Insufficient developmental excitatory neuronal activity fails to foster establishment of normal levels of inhibitory neuronal activity. Int $\mathrm{J}$ Dev Neurosci 2016; 55: 66-71.

[http://dx.doi.org/10.1016/j.ijdevneu.2016.09.009] [PMID: 27686511]

[43] Zemianek JM, Lee S, Guaraldi M, Shea TB. Accelerated establishment of mature signaling patterns following stimulation of developing neuronal networks: "learning" versus "plasticity". Int J Dev Neurosci 2012; 30(7): 602-6.

[http://dx.doi.org/10.1016/j.ijdevneu.2012.08.001] [PMID: 22906544]

[44] Buonomano DV, Merzenich MM. Cortical plasticity: From synapses to maps. Annu Rev Neurosci 1998; 21: 149-86.

[http://dx.doi.org/10.1146/annurev.neuro.21.1.149] [PMID: 9530495]

[45] Maren S, Baudry M. Properties and mechanisms of long-term synaptic plasticity in the mammalian brain: Relationships to learning and memory. Neurobiol Learn Mem 1995; 63(1): 1-18. [http://dx.doi.org/10.1006/nlme.1995.1001] [PMID: 7663875] 
[46] Malinow R, Malenka RC. AMPA receptor trafficking and synaptic plasticity. Annu Rev Neurosci 2002; 25: 103-26.

[http://dx.doi.org/10.1146/annurev.neuro.25.112701.142758] [PMID: 12052905]

[47] Poirazi P, Mel BW. Impact of active dendrites and structural plasticity on the memory capacity of neural tissue. Neuron 2001; 29(3): 779-96. [http://dx.doi.org/10.1016/S0896-6273(01)00252-5] [PMID 11301036]

[48] Bruel-Jungerman E, Davis S, Laroche S. Brain plasticity mechanisms and memory: A party of four. Neuroscientist 2007; 13(5): 492-505. [http://dx.doi.org/10.1177/1073858407302725] [PMID: 17901258]

[49] Neves G, Cooke SF, Bliss TV. Synaptic plasticity, memory and the hippocampus: A neural network approach to causality. Nat Rev Neurosci 2008; 9(1): 65-75.

[http://dx.doi.org/10.1038/nrn2303] [PMID: 18094707]

[50] Le Roux N, Amar M, Fossier P. Acquiring new information in a neuronal network: From Hebb's concept to homeostatic plasticity. J Soc Biol 2008; 202(2): 143-60.

[http://dx.doi.org/10.1051/jbio:2008018] [PMID: 18547512]

[51] Stegenga J, Le Feber J, Marani E, Rutten WL. The effect of learning on bursting. IEEE Trans Biomed Eng 2009; 56(4): 1220-7. [http://dx.doi.org/10.1109/TBME.2008.2006856] [PMID: 19272893]

[52] Rozov AV, Valiullina FF, Bolshakov AP. Mechanisms of long-term plasticity of hippocampal gabaergic synapses. Biochemistry (Mosc) 2017; 82(3): 257-63.

[http://dx.doi.org/10.1134/S0006297917030038] [PMID: 28320266]

[53] Castillo PE, Chiu CQ, Carroll RC. Long-term plasticity at inhibitory synapses. Curr Opin Neurobiol 2011; 21(2): 328-38.

[http://dx.doi.org/10.1016/j.conb.2011.01.006] [PMID: 21334194]

[54] Feldman DE. Synaptic mechanisms for plasticity in neocortex. Annu Rev Neurosci 2009; 32: 33-55.

[http://dx.doi.org/10.1146/annurev.neuro.051508.135516] [PMID: 19400721]

[55] Maffei A. The many forms and functions of long term plasticity at GABAergic synapses. Neural Plast 2011; 2011254724

[http://dx.doi.org/10.1155/2011/254724] [PMID: 21789285]

[56] Nelson SB, Turrigiano GG. Strength through diversity. Neuron 2008; 60(3): 477-82

[http://dx.doi.org/10.1016/j.neuron.2008.10.020] [PMID: 18995822]

[57] Shultz AM, Lee S, Guaraldi M, Shea TB, Yanco HC. Robot-embodied neuronal networks as a model for learning. Open Neurol J 2017; 11 : 39-47.

[http://dx.doi.org/10.2174/1874205X01711010039] [PMID: 29151990]

[58] Demarse TB, Wagenaar DA, Blau AW, Potter SM. The neurally controlled animat: Biological brains acting with simulated bodies. Auton Robots 2001; 11(3): 305-10.

[http://dx.doi.org/10.1023/A:1012407611130] [PMID: 18584059]

[59] Novellino A, D'Angelo P, Cozzi L, Chiappalone M. Connecting neurons to a mobile robot: An in vitro bidirectional neural interface Comp Intell Neurosci 2007.

[http://dx.doi.org/10.1155/2007/12725]

[60] Budd GE. Early animal evolution and the origins of nervous systems. Philos Trans R Soc Lond B Biol Sci 2015; 370(1684)20150037 [http://dx.doi.org/10.1098/rstb.2015.0037] [PMID: 26554037]

[61] Miljkovic-Licina M, Gauchat D, Galliot B. Neuronal evolution: Analysis of regulatory genes in a first-evolved nervous system, the hydra nervous system. Biosystems 2004; 76(1-3): 75-87.

[http://dx.doi.org/10.1016/j.biosystems.2004.05.030] [PMID: 15351132]

[62] Josipovic Z. Nondual awareness: Consciousness-as-such as nonrepresentational reflexivity. Prog Brain Res 2019; 244: 273-98. [http://dx.doi.org/10.1016/bs.pbr.2018.10.021] [PMID: 30732841]

[63] León-Domínguez U, León-Carrión J. Prefrontal neural dynamics in consciousness. Neuropsychologia 2019; 131: 25-41.

[http://dx.doi.org/10.1016/j.neuropsychologia.2019.05.018] [PMID: 31132421]

[64] Hutchinson BT. Toward a theory of consciousness: A review of the neural correlates of inattentional blindness. Neurosci Biobehav Rev
2019; 104: 87-99

[http://dx.doi.org/10.1016/j.neubiorev.2019.06.003]

[PMID: 31173773]

[65] Demertzi A, Soddu A, Laureys S. Consciousness supporting networks. Curr Opin Neurobiol 2013; 23(2): 239-44.

[http://dx.doi.org/10.1016/j.conb.2012.12.003] [PMID: 23273731]

[66] Katz PS. Evolution of central pattern generators and rhythmic behaviours. Philos Trans R Soc Lond B Biol Sci 2016; 371(1685)20150057

[http://dx.doi.org/10.1098/rstb.2015.0057] [PMID: 26598733]

[67] Gazzaniga MS. Brain mechanisms and conscious experience. Ciba Found Symp 1993; 174: 247-57

[PMID: 8391417]

[68] Rees G, Kreiman G, Koch C. Neural correlates of consciousness in humans. Nat Rev Neurosci 2002; 3(4): 261-70. [http://dx.doi.org/10.1038/nrn783] [PMID: 11967556]

[69] Guldenmund P, Vanhaudenhuyse A, Boly M, Laureys S, Soddu A. A default mode of brain function in altered states of consciousness. Arch Ital Biol 2012; 150(2-3): 107-21. [PMID: 23165872]

[70] Merker B. Consciousness without a cerebral cortex: A challenge for neuroscience and medicine. Behav Brain Sci 2007; 30(1): 63-81. [http://dx.doi.org/10.1017/S0140525X07000891] [PMID: 17475053]

[71] Laureys S. The neural correlate of (un)awareness: Lessons from the vegetative state. Trends Cogn Sci 2005; 9(12): 556-9. [http://dx.doi.org/10.1016/j.tics.2005.10.010] [PMID: 16271507]

[72] Lee S, Zemianek J, Shea TB. Rapid, reversible impairment of synaptic signaling in cultured cortical neurons by exogenously-applied amyloid- $\beta$. J Alzheimers Dis 2013; 35(2): 395-402. [http://dx.doi.org/10.3233/JAD-122452] [PMID: 23403533]

[73] Lee S, Sohal IS, Therrien MA, Pal AK, Bello D, Shea TB. Additive impairment of synaptic signaling in cultured cortical neurons by exogenously-applied oligomerized amyloid- $\beta$ and airborne nanoparticles generated during photocopying. J Alzheimers Dis 2015; 47(1): 49-54.

[http://dx.doi.org/10.3233/JAD-150099] [PMID: 26402753]

[74] Taddeo MA, Lee S, Shea TB. Synergistic inhibition of synaptic signaling in cortical cultures by subcytotoxic levels of oligomerized amyloid- $\beta$ and iron: Alleviation by zinc. J Alzheimers Dis 2014; 41(2): 365-9.

[http://dx.doi.org/10.3233/JAD-132696] [PMID: 24625798]

[75] Guaraldi M, Lee S, Shea TB. Synaptic signals from glutamate-treated neurons induce aberrant post-synaptic signals in untreated neuronal networks. Open Neurol J 2020; 14: 59.

[http://dx.doi.org/10.2174/1874205X02014010059]

[76] Sevush S. Single-neuron theory of consciousness. J Theor Biol 2006; 238(3): 704-25.

[http://dx.doi.org/10.1016/j.jtbi.2005.06.018] [PMID: 16083912]

[77] Shultz A, Lee S, Shea TB, Yanco H. Biological and simulated neuronal networks show similar competence on a visual tracking task. Proceed. $5^{\text {th }}$ Int Conf on Devel and Learning, Epigenetic Robotics. (ICDL-EPIROB 2015).

[78] Hales CM, Rolston JD, Potter SM. How to culture, record and stimulate neuronal networks on micro-electrode arrays (MEAs). J Vis Exp 2010; 39(39)e2056

[http://dx.doi.org/10.3791/2056] [PMID: 20517199]

[79] Mahmud M, Bertoldo A, Girardi S, Maschietto M, Vassanelli S. SigMate: A MATLAB-based neuronal signal processing tool. Annu Int Conf IEEE Eng Med Biol Soc 2010; 2010: 1352-5. [http://dx.doi.org/10.1109/IEMBS.2010.5626747] [PMID: 21096329]

[80] Garma LD, Matino L, Melle G, et al. Cost-effective and multifunctional acquisition system for in vitro electrophysiological investigations with multi-electrode arrays PLoS One 2019; 14(3)e 0214017

[http://dx.doi.org/10.1371/journal.pone.0214017] [PMID: 30908502]

[81] Chen R. Interactions between inhibitory and excitatory circuits in the human motor cortex. Exp Brain Res 2004; 154(1): 1-10. [http://dx.doi.org/10.1007/s00221-003-1684-1] [PMID: 14579004] 